\title{
Some Correlation Inequalities for Ising Antiferromagnets
}

\author{
David Klein \\ Department of Mathematics \\ California State University \\ Northridge, California 91330 \\ USA
}

\author{
Wei-Shih Yang \\ Department of Mathematics \\ University of Colorado \\ Colorado Springs, Colorado 80933 \\ USA
}

Abstract. We prove some inequalities for two-point correlations of Ising antiferromagnets and derive inequalities relating correlations of ferromagnets to correlations of antiferromagnets whose interactions and field strengths have equal magnitudes. The proofs are based on the method of duplicate spin variables introduced by J. Percus and used by several authors (Refs. 3-8) to derive correlation inequalities for Ising ferromagnets.

\section{Introduction}

Correlation inequalities have played an important role in statistical mechanics, especially as applied to ferromagnetic Hamiltonians. It is the purpose of this note to apply known techniques to obtain some correlation inequalities for antiferromagnets.

Let $\mathrm{H}_{1}(\sigma)$ be a ferromagnetic Hamiltonian for finite volume $\Lambda$ in $\mathbf{Z}^{\mathrm{d}}$ given by

$$
\mathrm{H}_{1}(\sigma)=\sum_{(\mathrm{i}, \mathrm{j}) \subset \Lambda} \mathrm{J}_{\mathrm{ij}} \sigma_{\mathrm{i}} \sigma_{\mathrm{j}}+\sum_{\mathrm{i} \in \Lambda} \mathrm{J}_{\mathrm{ij}} \sigma_{\mathrm{i}} \bar{\sigma}_{\mathrm{j}}-\mathrm{h} \sum_{\mathrm{i} \in \Lambda} \sigma_{\mathrm{i}}
$$

and $\mathrm{H}(\mathrm{x})$ a corresponding antiferromagnetic Hamiltonian for $\Lambda$ given by

$$
H(x)=\sum_{(i, j) \subset \Lambda} K_{i j} x_{i} x_{j}+\sum_{\substack{i \in \Lambda \\ j \notin \Lambda}} K_{i j} x_{i} \bar{x}_{j}-h \sum_{i \in \Lambda} x_{i}
$$

where the first sums in (1.1) and (1.2) are over all distinct pairs (i,j) in $\Lambda,\left\{\bar{x}_{j}\right\}$ and $\left\{\bar{\sigma}_{j}\right\}$ represent boundary configurations, and $\mathrm{K}_{\mathrm{ij}}=(-1)|\mathrm{i}|+|\mathrm{j}| \mathrm{J}_{\mathrm{ij}}$. Here $|\mathrm{i}|=\left|\left(\mathrm{i}_{1}, \mathrm{i}_{2}, \ldots, \mathrm{i}_{\mathrm{d}}\right)\right|=$ $\left|\mathrm{i}_{1}\right|+\cdots+\left|\mathrm{i}_{\mathrm{d}}\right|$ and $\mathrm{x}_{\mathrm{i}}, \sigma_{\mathrm{i}}= \pm 1$, and $\mathrm{J}_{\mathrm{ij}} \leq 0$. We will consider (1.2) with the change of variable $\mathrm{x}_{\mathrm{i}}=(-1)^{|\mathrm{i}|} \mathrm{s}_{\mathrm{i}}$ and denote the resulting Hamiltonian by $\mathrm{H}_{2}(\mathrm{~s})$ so that 


$$
\mathrm{H}_{2}(\mathrm{~s})=\sum_{(\mathrm{i}, \mathrm{j}) \subset \Lambda} \mathrm{J}_{\mathrm{ij}} \mathrm{s}_{\mathrm{i}} \mathrm{s}_{\mathrm{j}}+\sum_{\substack{\mathrm{i} \in \Lambda \\ \mathrm{j} \notin \Lambda}} \mathrm{J}_{\mathrm{ij}} \mathrm{s}_{\mathrm{i}} \overline{\mathrm{s}}_{\mathrm{j}}-\sum_{\mathrm{i} \in \Lambda} \mathrm{k}_{\mathrm{i}} \mathrm{s}_{\mathrm{i}}
$$

where $\mathrm{k}_{\mathrm{i}}=(-1)^{|\mathrm{i}|} \mathrm{h}$. We will denote expectations with respect to the finite volume Gibbs states corresponding to (1.1) and (1.3) by $\langle\cdot\rangle_{\mathrm{F}}$ and $\langle\cdot\rangle_{\mathrm{A}}$ respectively; boundary configurations will always be assumed fixed.

In Section 2 of this paper we derive Lebowitz-type inequalities ${ }^{3}$ which allow the comparison of correlations corresponding to Hamiltonians (1.1) and (1.3). When $\mathrm{h}=0$ (1.1) and (1.3) are equal and have equal correlation functions. When $h \neq 0 \mathrm{H}_{1}(\sigma)$ has a unique phase for all temperatures, which implies decay properties of truncated correlation functions for $\mathrm{H}_{1}(\sigma)$. Our inequalities are valid for all $h$, even though $h \neq 0$ includes both single and multiple phase regions for $\mathrm{H}(\mathrm{x})$ (see, for example, Ref. 1).

In Section 3 we prove some monotonicity properties for two-point correlations corresponding to (1.3). The method of proof is based on the techniques used by Messager and Miracle-Sole ${ }^{4}$ to derive, among other things, monotonicity properties for correlations corresponding to nearest neighbor ferromagnetic interactions. We make some modifications of their methods to accommodate nonnearest neighbor interactions and nonpositive external fields $\left\{\mathrm{k}_{\mathrm{i}}\right\}$. We allow our Hamiltonians to have infinite range, but our inequalities are weaker than those of Ref. 4 for the ferromagnetic case. We note that Hegerfeldt ${ }^{2}$ generalized some of the monotonicity results in Ref. 4 for ferromagnetic correlations, but the methods of Ref. 2 do not seem to extend readily to antiferromagnetic interactions. 


\section{Comparison of Correlations}

Let two Ising spin Hamiltonians $\mathrm{H}_{\mathrm{a}}(\sigma)$ and $\mathrm{H}_{\mathrm{b}}(\mathrm{s})$ for volume $\Lambda$ in $\mathbf{Z}^{\mathrm{d}}$ be given by

$$
\begin{aligned}
& \mathrm{H}_{\mathrm{a}}(\sigma)=\sum_{(\mathrm{i}, \mathrm{j}) \subset \Lambda} \mathrm{J}_{\mathrm{ij}} \sigma_{\mathrm{i}} \sigma_{\mathrm{j}}-\sum_{\mathrm{i} \in \Lambda} \mathrm{h}_{\mathrm{i}} \sigma_{\mathrm{i}} \\
& \mathrm{H}_{\mathrm{b}}(\mathrm{s})=\sum_{(\mathrm{i}, \mathrm{j}) \subset \Lambda} \mathrm{J}_{\mathrm{ij}} \mathrm{s}_{\mathrm{i}} \mathrm{s}_{\mathrm{j}}-\sum_{\mathrm{i} \in \Lambda} \mathrm{k}_{\mathrm{i}} \mathrm{s}_{\mathrm{i}}
\end{aligned}
$$

where $\mathrm{J}_{\mathrm{ij}} \leq 0$ and $\sigma_{\mathrm{i}}, \mathrm{s}_{\mathrm{i}}= \pm 1$ for all $\mathrm{i}, \mathrm{j} \in \mathbf{Z}^{\mathrm{d}}$. The external field variables $\mathrm{h}_{\mathrm{i}}$ and $\mathrm{k}_{\mathrm{i}}$ are of the form

$$
\begin{aligned}
& \mathrm{h}_{\mathrm{i}}=\mathrm{h}_{\mathrm{i}}^{\prime}-\sum_{\mathrm{j} \notin \Lambda} \mathrm{J}_{\mathrm{ij}} \overline{\mathrm{\sigma}}_{\mathrm{j}} \\
& \mathrm{k}_{\mathrm{i}}=\mathrm{k}_{\mathrm{i}}^{\prime}-\sum_{\mathrm{j} \notin \Lambda} \mathrm{J}_{\mathrm{ij}} \overline{\mathrm{s}}_{\mathrm{j}}
\end{aligned}
$$

where $\left\{\bar{\sigma}_{\mathrm{j}}\right\}$ and $\left\{\overline{\mathrm{s}}_{\mathrm{j}}\right\}$ may be interpreted as fixed boundary configurations. Correlation functions, for a finite set $\mathrm{B}$ in $\mathbf{Z}^{\mathrm{d}}$, with respect to the finite volume Gibbs measures for (2.1) and (2.2) will be denoted by $\left\langle\prod_{\mathrm{i} \in \mathrm{B}} \sigma_{\mathrm{i}}\right\rangle \equiv\left\langle\sigma_{\mathrm{B}}\right\rangle$ and $\left\langle\prod_{\mathrm{i} \in \mathrm{B}} \mathrm{s}_{\mathrm{i}}\right\rangle \equiv\left\langle\mathrm{s}_{\mathrm{B}}\right\rangle$ respectively.

Let

$$
\mathrm{H}_{\mathrm{i}}=\mathrm{h}_{\mathrm{i}}+\mathrm{k}_{\mathrm{i}} \quad \text { and } \quad \mathrm{K}_{\mathrm{i}}=\mathrm{h}_{\mathrm{i}}-\mathrm{k}_{\mathrm{i}} \text {. }
$$

Define spin variables $\mathrm{q}_{\mathrm{i}}$ and $\mathrm{t}_{\mathrm{i}}$ taking values $-1,0,+1$ by

$$
\mathrm{t}_{\mathrm{i}}=1 / 2\left(\sigma_{\mathrm{i}}+\mathrm{s}_{\mathrm{i}}\right) \text { and } \mathrm{q}_{\mathrm{i}}=1 / 2\left(\sigma_{\mathrm{i}}-\mathrm{s}_{\mathrm{i}}\right)
$$

Let $\langle\langle\cdot\rangle\rangle$ denote expectations with respect to the product measure

$$
\mu(\sigma, \mathrm{s})=\frac{1}{\mathrm{Z}_{\mathrm{a}}(\Lambda)} \frac{1}{\mathrm{Z}_{\mathrm{b}}(\Lambda)} \exp \left\{-\beta\left[\mathrm{H}_{\mathrm{a}}(\sigma)+\mathrm{H}_{\mathrm{b}}(\mathrm{s})\right]\right\}
$$


where $\mathrm{Z}_{\mathrm{a}}(\Lambda)$ and $\mathrm{Z}_{\mathrm{b}}(\Lambda)$ are the partition functions for $\mathrm{H}_{\mathrm{a}}(\sigma)$ and $\mathrm{H}_{\mathrm{b}}(\Lambda)$ respectively. For finite sets $\mathrm{A}, \mathrm{B}$ in $\mathbf{Z}^{\mathrm{d}}$, let $\mathrm{t}_{\mathrm{A}}=\prod_{\mathrm{i} \in \mathrm{A}} \mathrm{t}_{\mathrm{i}}$ and $\mathrm{s}_{\mathrm{B}}=\prod_{\mathrm{i} \in \mathrm{B}} \mathrm{q}_{\mathrm{i}}$. The following theorem, though not stated in this generality, was proved by Lebowitz in Ref. 3 (see also Percus ${ }^{5}$ and Sylvester ${ }^{7}$.

Theorem 2.1 If $\mathrm{H}_{\mathrm{i}}, \mathrm{K}_{\mathrm{i}} \geq 0$ for all $\mathrm{i} \in \Lambda$, then for any two subsets $\mathrm{A}$, B in $\Lambda$,
a) $\left\langle\left\langle\mathrm{t}_{\mathrm{A}}\right\rangle\right\rangle,\left\langle\left\langle\mathrm{q}_{\mathrm{A}}\right\rangle\right\rangle \geq 0$
b) $\left\langle\left\langle\mathrm{t}_{\mathrm{A}} \mathrm{t}_{\mathrm{B}}\right\rangle\right\rangle \geq\left\langle\left\langle\mathrm{t}_{\mathrm{A}}\right\rangle\right\rangle\left\langle\left\langle\mathrm{t}_{\mathrm{B}}\right\rangle\right\rangle$
c) $\left\langle\left\langle\mathrm{q}_{\mathrm{A}} \mathrm{q}_{\mathrm{B}}\right\rangle\right\rangle \geq\left\langle\left\langle\mathrm{q}_{\mathrm{A}}\right\rangle\right\rangle\left\langle\left\langle\mathrm{q}_{\mathrm{B}}\right\rangle\right\rangle$
d) $\left\langle\left\langle\mathrm{q}_{\mathrm{A}}\right\rangle\right\rangle\left\langle\left\langle\mathrm{t}_{\mathrm{B}}\right\rangle\right\rangle \geq\left\langle\left\langle\mathrm{q}_{\mathrm{A}} \mathrm{t}_{\mathrm{B}}\right\rangle\right\rangle$.

Remark 2.1 By symmetry, it may be assumed that $\mathrm{H}_{\mathrm{i}}, \mathrm{K}_{\mathrm{i}} \leq 0$, in which case inequalities a) - d) are modified by replacing each $q_{i}$ by $-q_{i}$ and each $t_{i}$ by $-t_{i}$.

Corollary 2.1 With the same assumptions as in Theorem 2.1,
a) $\left\langle\left\langle\mathrm{t}_{\mathrm{A}}\right\rangle\right\rangle$ decreases and $\left\langle\left\langle\mathrm{q}_{\mathrm{A}}\right\rangle\right\rangle$ increases as each $\mathrm{K}_{\mathrm{i}}$ increases
b) $\left\langle\left\langle t_{A}\right\rangle\right\rangle$ increases and $\left\langle\left\langle q_{A}\right\rangle\right\rangle$ decreases as each $H_{i}$ increases.

proof. This follows by differentiating $\left\langle\left\langle\mathrm{t}_{\mathrm{A}}\right\rangle\right\rangle$ and $\left\langle\left\langle\mathrm{q}_{\mathrm{A}}\right\rangle\right\rangle$ by $\mathrm{H}_{\mathrm{i}}$ or $\mathrm{K}_{\mathrm{i}}$ and applying $\mathrm{b}$ ), c), or d) of Theorem 2.1.

A substantial generalization of part a) of the following Corollary was proved by Lebowitz in Ref. 8 (see also Griffiths ${ }^{9}$ ).

Corollary 2.2 If $\mathrm{H}_{\mathrm{i}}, \mathrm{K}_{\mathrm{i}} \geq 0$ for all $\mathrm{i} \in \Lambda$, then for any subset $\mathrm{B}$ in $\Lambda$, and any $\mathrm{i}, \mathrm{j} \in \Lambda$,
a) $\left\langle\sigma_{\mathrm{B}}\right\rangle \geq\left|\left\langle\mathrm{s}_{\mathrm{B}}\right\rangle\right|$
b) $\left\langle\sigma_{i} \sigma_{j}\right\rangle-\left\langle\sigma_{i}\right\rangle\left\langle\sigma_{j}\right\rangle \leq\left\langle s_{i} s_{j}\right\rangle-\left\langle s_{i}\right\rangle\left\langle s_{i}\right\rangle$ 
proof. The following identities, where $\sigma_{\mathrm{i}}$ and $\mathrm{s}_{\mathrm{i}}$ may be complex numbers are well known and easily verified (see for example Ref. 2):

$$
\begin{aligned}
& \prod_{i \in A} \sigma_{i}+\prod_{i \in A} s_{i}=2^{-|A|+1} \sum_{\substack{B \in A \\
|B| e v e n}} \prod_{i \in B}\left(\sigma_{i}-s_{i}\right) \prod_{i \in A \backslash B}\left(\sigma_{i}+s_{i}\right) \\
& \prod_{i \in A} \sigma_{i}-\prod_{i \in A} s_{i}=2^{-|A|+1} \sum_{\substack{B \subset A \\
|B| \text { odd }}} \prod_{i \in B}\left(\sigma_{i}-s_{i}\right) \prod_{i \in A \mid B}\left(\sigma_{i}+s_{i}\right)
\end{aligned}
$$

where $|\mathrm{A}|$ denotes the cardinality of A. Identifying $\sigma_{\mathrm{i}}$ and $\mathrm{s}_{\mathrm{i}}$ as Ising spin variables yields,

$$
\prod_{i \in A} \sigma_{i}-\prod_{i \in A} s_{i}=2 \sum_{\substack{B \subset A \\|B| \text { odd }}} q_{B} t_{A \mid B}
$$

and

$$
\prod_{i \in A} \sigma_{i}+\prod_{i \in A} s_{i}=2 \sum_{\substack{B \subset A \\|B| e v e n}} q_{B} t_{A \backslash B}
$$

Taking $\langle\langle\cdot\rangle\rangle$ expectations of (2.10) and (2.11) yields part a) of the corollary. The proof of part b) follows directly from part d) of Theorem 2.1 with $A=\{i\}$ and $B=\{j\}$. This completes the proof.

We now consider the special case for the Hamiltonians (2.1) and (2.2) where in equation (2.3), $\mathrm{h}_{\mathrm{i}}^{\mathrm{i}} \equiv \mathrm{h}$ for all $\mathrm{i}$ and some constant $\mathrm{h}$, and in equation (2.4) $\mathrm{k}_{\mathrm{i}}^{\prime}=(-1)^{\mathrm{il}} \mathrm{h}$. With these identifications $\mathrm{H}_{\mathrm{a}}(\sigma)$ equals $\mathrm{H}_{1}(\sigma)$, given by equation (1.1), and $\mathrm{H}_{\mathrm{b}}(\mathrm{s})$ equals $\mathrm{H}_{2}(\mathrm{~s})$, 
given by equation (1.3). The following corollary is now an immediate consequence of Corollary 2.2.

Corollary 2.3 Let $h \geq 0$. Assume that for all $i \in \Lambda$

$$
\begin{aligned}
& \text { 1) } \mathrm{h} \geq 1 / 2 \sum_{\mathrm{j} \notin \Lambda} \mathrm{J}_{\mathrm{ij}}\left(\bar{\sigma}_{\mathrm{j}}+\overline{\mathrm{s}}_{\mathrm{j}}\right) \\
& \text { 2) } \sum_{\mathrm{j} \notin \Lambda} \mathrm{J}_{\mathrm{ij}}\left(\bar{\sigma}_{\mathrm{j}}-\overline{\mathrm{s}}_{\mathrm{j}}\right) \leq 0 .
\end{aligned}
$$

Then for any subset $\mathrm{B}$ of $\Lambda$, and any $\mathrm{i}, \mathrm{j} \in \Lambda$, the following inequalities for the correlations of the Hamiltonians given by (1.1) and (1.3) hold:
a) $\left\langle\prod_{\mathrm{i} \in \mathrm{B}} \sigma_{\mathrm{i}}\right\rangle_{\mathrm{F}} \geq\left|\left\langle\prod_{\mathrm{i} \in \mathrm{B}} \mathrm{s}_{\mathrm{i}}\right\rangle_{\mathrm{A}}\right|$
b) $\left\langle\sigma_{\mathrm{i}} \sigma_{\mathrm{j}}\right\rangle_{\mathrm{F}}-\left\langle\sigma_{\mathrm{i}}\right\rangle_{\mathrm{F}}\left\langle\sigma_{\mathrm{j}}\right\rangle_{\mathrm{F}} \leq\left\langle\mathrm{s}_{\mathrm{i}} \mathrm{s}_{\mathrm{j}}\right\rangle_{\mathrm{A}}-\left\langle\mathrm{s}_{\mathrm{i}}\right\rangle_{\mathrm{A}}\left\langle\mathrm{s}_{\mathrm{j}}\right\rangle_{\mathrm{A}}$.

Remark 2.2 An analogous statement may be made for $\mathrm{h} \leq 0$ (see Remark 2.1).

Remark 2.3 The hypotheses to Corollary 2.3 are satisfied, for example, if $\bar{\sigma}_{\mathrm{j}} \equiv+1$ for all $\mathrm{j}$ $\notin \Lambda$. In this case $\overline{\mathrm{s}}_{\mathrm{j}}$ for $\mathrm{j} \notin \Lambda$ may be chosen arbitrarily. It is also easily shown that if $\mathrm{H}_{1}(\sigma)$ and $\mathrm{H}_{2}$ (s) both have empty or both have periodic boundary conditions, then a) and b) of Corollary 2.3 hold.

\section{Monotonicity Properties for Antiferromagnets}

In this section we prove some monotonicity properties for two point correlations for antiferromagnets. Denote by H(s) the Hamiltonian,

$$
\mathrm{H}(\mathrm{s})=\sum_{\Lambda} \mathrm{J}_{\mathrm{ij}} \mathrm{s}_{\mathrm{i}} \mathrm{s}_{\mathrm{j}}-\sum_{\mathrm{i} \in \Lambda} \mathrm{k}_{\mathrm{i}} \mathrm{s}_{\mathrm{i}}
$$

where here and below $\sum_{\Lambda}$ means sum over all distinct pairs (i,j) in the subset $\Lambda$ in $\mathbf{Z}^{\mathrm{d}}$. We also assume that $\mathrm{J}_{\mathrm{ij}} \leq 0$ and that $\mathrm{J}_{\mathrm{ij}}$ is a function of $\| \mathrm{i}$ - $\mathrm{j} \|$, the Euclidean norm of $\mathrm{i}$ - $\mathrm{j}$. The external field $\mathrm{k}_{\mathrm{i}}$ is given by 


$$
\mathrm{k}_{\mathrm{i}}=\mathrm{k}_{\mathrm{i}}^{\prime}-\sum_{\mathrm{j} \notin \Lambda} \mathrm{J}_{\mathrm{ij}} \overline{\mathrm{s}}_{\mathrm{j}}
$$

for some boundary configuration $\left\{\bar{s}_{j}\right\}$, where $k_{i}^{\prime}=(-1)^{|i|} h$ for some $h \geq 0$, so that $H(s)$ is equal to the antiferromagnetic Hamiltonian (1.3). In this section, denote by \langle\rangle or $\langle\lambda$ expectations with respect to the finite volume Gibbs state determined by (3.1).

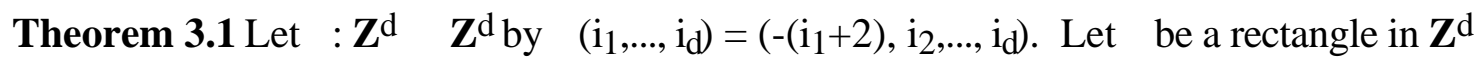
invariant under $\varphi$ and let the boundary configuration $\left\{\bar{s}_{j}\right\}$ be invariant under $\varphi$. Suppose also that $\left|\mathrm{J}_{\mathrm{ij}}\right| \geq 1 / 2\left|\mathrm{~J}_{\mathrm{i} \varphi(\mathrm{j})}\right|$. Then for any $\mathrm{i}, \mathrm{j} \in \Lambda$ with $\mathrm{i}_{1}, \mathrm{j}_{1} \geq 0$,

$$
\left\langle s_{i} s_{j}\right\rangle \geq\left\langle s_{i} s_{\varphi(j)}\right\rangle
$$

Remark 3.1 It is also possible to consider periodic boundary conditions. If $0 \in \Lambda$ (3.3) and the symmetry of the finite volume Gibbs State imply

$$
\left\langle\mathrm{s}_{0} \mathrm{~s}_{\mathrm{j}}\right\rangle \geq\left\langle\mathrm{s}_{0} \mathrm{~s}_{\left(\mathrm{j}_{1}+2, \mathrm{j}_{2}, \ldots, \mathrm{j}_{\mathrm{d}}\right)}\right\rangle
$$

for $\mathrm{j}_{1} \geq 0$, where $\mathrm{s}_{0}$ is the spin at the origin of $\mathbf{Z}^{\mathrm{d}}$.

proof. Let

$$
\begin{aligned}
& \Lambda_{+}=\left\{i \in \Lambda: i_{1}>-1\right\} \\
& \Lambda_{0}=\left\{i \in \Lambda: i_{1}=-1\right\} \\
& \Lambda_{-}=\left\{i \in \Lambda: i_{1}<-1\right\} .
\end{aligned}
$$

Then $\Lambda=\Lambda_{+} \cup \Lambda_{0} \cup \Lambda_{-}$and $\varphi\left(\Lambda_{+}\right)=\Lambda_{-}, \varphi\left(\Lambda_{-}\right)=\Lambda_{+}$, and $\varphi\left(\Lambda_{0}\right)=\Lambda_{0}$. Denote $\varphi(\mathrm{i})$ by $\mathrm{i} \sim$. With this notation we can write, 


$$
\begin{aligned}
\Lambda \Sigma_{i j} s_{i} s_{j}= & \sum_{\Lambda+} J_{i j}\left(s_{i} s_{j}+s_{i} \sim s_{j} \sim\right)+\sum_{i \in \Lambda o} \sum_{j \in \Lambda+} J_{i j}\left(s_{i} s_{j}+s_{i} \sim s_{j}\right) \\
& +1 / 2 \sum_{\Lambda o} J_{i j}\left(s_{i} s_{j}+s_{i} \sim s_{j}\right)+1 / 2 \sum_{\Lambda+} J_{i j \sim}\left(s_{i} s_{j} \sim+s_{i \sim} s_{j}\right) \\
& +1 / 2 \sum_{i \in \Lambda+} J_{i i \sim}\left(s_{i} s_{i} \sim+s_{i} s_{i}\right) .
\end{aligned}
$$

The last two terms on the right side of (3.5) may be rewritten as,

$$
\begin{aligned}
& 1 / 2 \sum_{\Lambda+} \mathrm{J}_{\mathrm{ij} \sim}\left(\mathrm{s}_{\mathrm{i}}+\mathrm{s}_{\mathrm{i} \sim}\right)\left(\mathrm{s}_{\mathrm{j}}+\mathrm{s}_{\mathrm{j} \sim}\right)-1 / 2 \sum_{\Lambda+} \mathrm{J}_{\mathrm{ij} \sim}\left(\mathrm{s}_{\mathrm{i}} \mathrm{s}_{\mathrm{j}}+\mathrm{s}_{\left.\mathrm{i} \sim \mathrm{s}_{\mathrm{j} \sim}\right)}\right. \\
& +1 / 2 \sum_{\mathrm{i} \in \Lambda_{+}} \mathrm{J}_{\mathrm{ii} \sim}\left(\mathrm{s}_{\mathrm{i}}+\mathrm{s}_{\mathrm{i} \sim}\right)^{2}-\sum_{\mathrm{i} \in \Lambda_{+}} \mathrm{J}_{\mathrm{ii} \sim} .
\end{aligned}
$$

Let

$$
\mathrm{t}_{\mathrm{i}}=1 / 2\left(\mathrm{~s}_{\mathrm{i}}+\mathrm{s}_{\mathrm{i} \sim}\right) \text { and } \mathrm{q}_{\mathrm{i}}=1 / 2\left(\mathrm{~s}_{\mathrm{i}}-\mathrm{s}_{\mathrm{i} \sim}\right)
$$

so that

$$
s_{i} s_{j}+s_{i \sim} s_{j \sim}=2\left(t_{i} t_{j}+q_{i} q_{j}\right) .
$$

Combining (3.5) - (3.7) and observing that $\mathrm{q}_{i}=0$ if $\mathrm{i} \in \Lambda_{0}$ gives,

$$
\begin{aligned}
\sum_{\Lambda} \mathrm{J}_{\mathrm{ij}} \mathrm{s}_{\mathrm{i}} \mathrm{s}_{\mathrm{j}}= & \sum_{\Lambda+}\left(2 \mathrm{~J}_{\mathrm{ij}}-\mathrm{J}_{\mathrm{ij} \sim}\right) \mathrm{q}_{\mathrm{i}} \mathrm{q}_{\mathrm{j}}+\sum_{\Lambda+} 2\left(\mathrm{~J}_{\mathrm{ij}}+\mathrm{J}_{\mathrm{ij} \sim}\right) \mathrm{t}_{\mathrm{i}} \mathrm{t}_{\mathrm{j}} \\
& +2 \sum_{\mathrm{i} \in \Lambda \mathrm{o}} \sum_{\mathrm{j} \in \Lambda_{+}} \mathrm{J}_{\mathrm{ij}} \mathrm{t}_{\mathrm{i}} \mathrm{t}_{\mathrm{j}}+\sum_{\Lambda \mathrm{o}} \mathrm{J}_{\mathrm{ij}} \mathrm{t}_{\mathrm{i}} \mathrm{t}_{\mathrm{j}} \\
& +2 \sum_{\Lambda+} \mathrm{J}_{\mathrm{ii} \sim \mathrm{t}} \mathrm{t}_{\mathrm{i}}^{2}-\sum_{\Lambda+} \mathrm{J}_{\mathrm{ii} \sim} .
\end{aligned}
$$

Now define $\mathrm{H}_{\mathrm{i}}=\mathrm{k}_{\mathrm{i}}+\mathrm{k}_{\mathrm{i} \sim}$ and $\mathrm{K}_{\mathrm{i}}=\mathrm{k}_{\mathrm{i}}-\mathrm{k}_{\mathrm{i} \sim}$ so that

$$
\mathrm{k}_{\mathrm{i}} \mathrm{s}_{\mathrm{i}}+\mathrm{k}_{\mathrm{i} \sim} \mathrm{s}_{\mathrm{i} \sim}=\mathrm{H}_{\mathrm{i}} \mathrm{t}_{\mathrm{i}}+\mathrm{K}_{\mathrm{i}} \mathrm{q}_{\mathrm{i}} \text {. }
$$

From the definition of $\varphi$ and $k_{i}$ and the invariance of the boundary conditions $\left\{s_{j}\right\}$ under $\varphi$, it follows that $\mathrm{K}_{\mathrm{i}}=0$ for all $\mathrm{i} \in \Lambda$. Thus to within an additive constant,

$$
\begin{aligned}
& H(s)=H^{1}(q)+H^{2}(t), \text { where } \\
& H^{1}(q)=\sum_{\Lambda+} N_{i j} q_{i} q_{j} \\
& H^{2}(t)=\sum_{\Lambda+\cup \Lambda o} M_{i j} t_{i} t_{j}+2 \sum_{i \in \Lambda+} J_{i i \sim} t_{i}^{2}+\sum_{i \in \Lambda+} H_{i} t_{i}+1 / 2 \sum_{i \in \Lambda o} H_{i} t_{i}
\end{aligned}
$$

and $\mathrm{N}_{\mathrm{ij}}$ and $\mathrm{M}_{\mathrm{ij}}$ are nonpositive. 
From the definitions of $q_{i}$ and $t_{i}$ it follows that $t_{i}=0$ iff $q_{i}= \pm 1$ and $q_{i}=0$ iff $t_{i}= \pm 1$. Also if $i \in \Lambda_{0}$, then $t_{i}= \pm 1$. For any functions $\phi(q)$ and $\psi(t)$,

$$
\langle\phi(\mathrm{q}) \psi(\mathrm{t})\rangle=\frac{1}{\mathrm{Z}_{\Lambda}(\mathrm{s})} \sum_{(\mathrm{q}, \mathrm{t})} \phi(\mathrm{q}) \psi(\mathrm{t}) \exp \left\{-\beta\left[\mathrm{H}^{1}(\mathrm{q})+\mathrm{H}^{2}(\mathrm{t})\right]\right\}
$$

where the sum in (3.11) is over all pairs $q=\left\{q_{i}\right\}_{i \in \Lambda+}$ and $t=\left\{t_{i}\right\}_{i \in \Lambda+} \cup \Lambda_{o}$. such that $\mathrm{t}_{\mathrm{i}}= \pm 1$ if $\mathrm{i} \in \Lambda_{0}$, and $\mathrm{q}_{\mathrm{i}}=0$ iff $\mathrm{t}_{\mathrm{i}}= \pm 1$ otherwise. Equation (3.11) may be rewritten as,

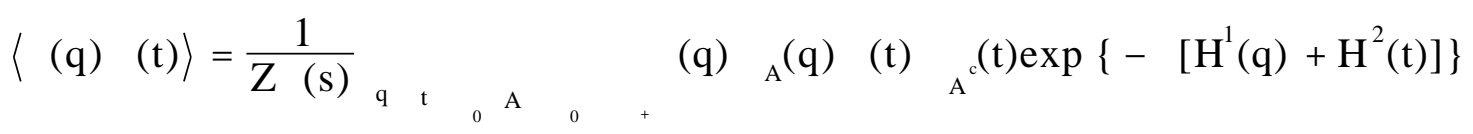

where the sums on $q$ and $t$ now include the values \pm 1 for $q_{i}$ and $t_{i}$, but not zero, $\mathrm{A}^{\mathrm{c}}=\left(\Lambda_{+} \cup \Lambda_{0}\right) \backslash \mathrm{A}$, and

$$
\chi_{A}(q)= \begin{cases}1, & \text { when } q_{i}=0 \text { if } \mathrm{f} i \in A \\ 0, & \text { otherwise }\end{cases}
$$

For any $\mathrm{A} \subset \Lambda_{0} \cup \Lambda_{+}$, let

$$
\begin{aligned}
P(A) & =\frac{\sum_{t} \chi_{A}{ }^{c}(t) \exp \left[-\beta H^{2}(t)\right] \sum_{q} \chi_{A}(q) \exp \left[-\beta H^{1}(q)\right]}{Z_{\Lambda}(s)} \\
& \equiv \frac{Z_{A}(t) Z_{A}{ }^{c}(q)}{Z_{\Lambda}(s)}
\end{aligned}
$$

where $\mathrm{Z}_{\mathrm{A}} \mathrm{c}(\mathrm{q})$ and $\mathrm{Z}_{\mathrm{A}}(\mathrm{t})$ are the usual Ising partition functions respectively for $\mathrm{H}^{1}(\mathrm{q})$ and $\mathrm{H}^{2}(\mathrm{t})$ with $\mathrm{q}_{\mathrm{i}}, \mathrm{t}_{\mathrm{i}}= \pm 1$. Then (3.12) may be rewritten as

$$
\langle\phi(\mathrm{q}) \psi(\mathrm{t})\rangle=\sum_{\Lambda_{0} \subset \mathrm{A} \subset \Lambda_{0} \cup \Lambda_{+}} \mathrm{P}(\mathrm{A})\left\langle\phi(\mathrm{q}) \chi_{\mathrm{A}}(\mathrm{q})\right\rangle_{\mathrm{A}^{\mathrm{c}}, \mathrm{q}}\left\langle\psi(\mathrm{t}) \chi_{\mathrm{A}^{\mathrm{c}}}(\mathrm{t})\right\rangle_{\mathrm{A}, \mathrm{t}}
$$


where $\left\langle\phi(\mathrm{q}) \chi_{\mathrm{A}}(\mathrm{q})\right\rangle_{\mathrm{A}^{\mathrm{c}}, \mathrm{q}}=\mathrm{Z}_{\mathrm{A}} \mathrm{c}(\mathrm{q})^{-1} \sum_{\mathrm{q}} \chi_{\mathrm{A}}(\mathrm{q}) \phi(\mathrm{q}) \exp \left[-\beta \mathrm{H}^{1}(\mathrm{q})\right]$ and $\left\langle\psi(\mathrm{t}) \chi_{\mathrm{A}} \mathrm{c}(\mathrm{t})\right\rangle_{\mathrm{A}, \mathrm{t}}$ has an analogous expression. Let $\psi(\mathrm{t}) \equiv 1$ and $\phi(\mathrm{q})=\mathrm{q}_{\mathrm{i}} \mathrm{q}_{\mathrm{j}}$. Then

$$
\left\langle\mathrm{q}_{\mathrm{i}} \mathrm{q}_{\mathrm{j}}\right\rangle=\sum_{\mathrm{A}} \mathrm{P}(\mathrm{A})\left\langle\mathrm{q}_{\mathrm{i}} \mathrm{q}_{\mathrm{j}} \chi_{\mathrm{A}}(\mathrm{q})\right\rangle_{\mathrm{A}^{\mathrm{c}}, \mathrm{q}} \geq 0
$$

since by Griffith's inequality each term in the sum is nonnegative. Thus

$$
\left\langle s_{i} s_{j}\right\rangle+\left\langle s_{i \sim} s_{j \sim}\right\rangle \geq\left\langle s_{i \sim} s_{j}\right\rangle+\left\langle s_{i} s_{j}\right\rangle
$$

and the conclusion of the theorem now follows from the invariance of $\Lambda$ and the boundary conditions under the reflection $\varphi$. This completes the proof.

The following corollary, establishing a version of the Percus inequality or first Lebowitz inequality, follows immediately from the arguments leading up to equation (3.14).

Corollary 3.1 With the hypotheses and notation of Theorem 3.1,

$$
\left\langle\prod_{i \in A}\left(s_{i}-s_{\phi(i)}\right\rangle \geq 0\right.
$$

for any $\mathrm{A}$ in $\Lambda_{+}$.

Theorem 3.2 Let $\Psi: \mathbf{Z}^{\mathrm{d}} \rightarrow \mathbf{Z}^{\mathrm{d}}$ by $\Psi\left(\mathrm{i}_{1}, \ldots, \mathrm{i}_{\mathrm{d}}\right)=\left(-\left(\mathrm{i}_{1}+1\right), \mathrm{i}_{2}, \ldots, \mathrm{i}_{\mathrm{d}}\right)$. Let a rectangle $\Lambda$ in $\mathbf{Z}^{\mathrm{d}}$ and a boundary condition $\left\{\bar{s}_{j}\right\}_{j \in \Lambda}$ c be invariant under $\Psi$. Then for any $i, j \in \Lambda$ with $\mathrm{i}_{1}, \mathrm{j}_{1} \geq 0$

$$
\left\langle s_{i} s_{j}\right\rangle \geq-\left\langle s_{i} s \Psi(j)\right\rangle
$$

The proof of Theorem 3.2 is similar to and simpler than the proof of Theorem 3.1. In this case $\Lambda_{+}=\{i \in \Lambda: i \geq 0\}, \Lambda_{-}=\{i \in \Lambda: i \leq-1\}$, and $\Lambda_{0}$ is empty. With analogous notation as in 
the proof of Theorem 3.1, $H_{i} \equiv 0$ and it follows that $\left\langle\prod_{i \in A} t_{i}\right\rangle \geq 0$ for any subset $A$ of $\Lambda_{+}$. The case in which $\mathrm{J}_{\mathrm{ij}}$ is nonzero only for $\| \mathrm{i}$ - $\mathrm{j} \|=1$ was essentially contained in the proof of an analogous theorem (Theorem 1) of Messager and Miracle-Sole ${ }^{4}$ for Ising ferromagnets.

Corollary 3.2 If $\mathrm{J}_{\mathrm{ij}}$ satisfies the conditions of Theorem 3.1, then

1) $\left\langle\mathrm{s}_{0} \mathrm{~s}_{\mathrm{j}}\right\rangle_{\infty}^{ \pm} \geq\left\langle\mathrm{s}_{0} \mathrm{~s}_{\left(\mathrm{j}_{1}+2, \mathrm{j}_{2}, \ldots, \mathrm{j}_{\mathrm{d}}\right.}\right\rangle_{\infty}^{ \pm}$

2) $\left\langle\mathrm{s}_{0} \mathrm{~s}_{\mathrm{j}}\right\rangle_{\infty}^{ \pm} \geq-\left\langle\mathrm{s}_{0} \mathrm{~s}_{\left(\mathrm{j}_{1}+1, \mathrm{j}_{2}, \ldots, \mathrm{j}_{\mathrm{d}} \mathrm{d}_{\infty}\right.}\right\rangle_{\infty}^{ \pm}$

for any j with $\mathrm{j}_{1} \geq 0$, where $\left\langle\mathrm{s}_{0} \mathrm{~s}_{\mathrm{j}}\right\rangle_{\infty}^{ \pm}=\lim _{\Lambda \uparrow \mathrm{Z}^{\mathrm{d}}}\left\langle\mathrm{s}_{0} \mathrm{~s}_{\mathrm{j}}\right\rangle_{\Lambda}$ with boundary conditions $\overline{\mathrm{s}}_{\mathrm{j}} \equiv+1$ or $\overline{\mathrm{s}}_{\mathrm{j}} \equiv$ -1 for all $\mathrm{j} \in \Lambda^{\mathrm{c}}$ and the limit may be taken along any sequence $\Lambda_{\mathrm{n}}$ increasing to $\mathbf{Z}^{\mathrm{d}}$.

proof. Let $\rho_{j}=1 / 2\left(s_{j}+1\right)$. Then $\rho_{0} \rho_{j}$ is an increasing function in the sense used in the FKG inequalities. Since $4 \rho_{0} \rho_{j}=\left[s_{0} s_{j}+s_{0}+s_{j}+1\right]$ and $s_{0}$ and $s_{j}$ are also increasing, it follows that $\lim \left\langle\mathrm{s}_{0} \mathrm{~s}_{\mathrm{j}}\right\rangle_{\Lambda}$ exists along any sequence $\Lambda_{\mathrm{n}}$ increasing to $\mathbf{Z}^{\mathrm{d}}$. Let $\Lambda_{\mathrm{n}}$ be as in Theorem 3.1 and let $\tilde{\Lambda}_{\mathrm{n}}$ be the reflection of $\Lambda_{\mathrm{n}}$ across the hyperplane $j_{1}=0$. Then

$$
\left\langle\mathrm{s}_{0} \mathrm{~s}_{\phi(\mathrm{j})}\right\rangle_{\Lambda_{\mathrm{n}}}=\left\langle\mathrm{s}_{0} \mathrm{~s}_{\left(\mathrm{j}_{1}+2, \mathrm{j}_{2}, \ldots, \mathrm{j}_{\mathrm{d}}{ }_{\mathrm{d}}\right.}\right\rangle_{X_{\mathrm{n}}}
$$

Inequality 1) now follows by applying Theorem 3.1 and taking limits. The proof of 2) is similar.

Remark 3.2 We note that other axes and reflections may be used in Theorems 3.1 and 3.2; the crucial point is that $\mathrm{H}_{\mathrm{i}}$ or $\mathrm{K}_{\mathrm{i}}$ or both (as in the case of ferromagnetic interactions) must be nonnegative (see (3.9)).

\section{Acknowledgement.}


One of us, Yang, was partially supported during the course of this research by NFS grant DMS 8902123.

\section{References}

1. Dobrushin, R. L.: Phase diagram of the two-dimensional Ising antiferromagnet (computer-assisted proof). Commun. Math. Phys. 102, 89-103 (1985)

2. Hegerfeldt, G. H.: Correlation inequalities for Ising ferromagnets with symmetries. Commun. Math. Phys. 57, 259-266 (1977)

3. Lebowitz, J. L.: GHS and other inequalities. Commun. Math. Phys. 35, 87-92 (1974)

4. Messager, A. and Miracle-Sole, S.: Correlation functions and boundary conditions in the Ising ferromagnet. J. Stat. Phys. 17 no. 4, 245-262 (1977)

5. Percus, J.: Correlation Inequalities for Ising spin lattices. Commun. Math. Phys. 40, 283308 (1975)

6. Schrader, R.: New correlation inequalities for the Ising model and $\mathrm{P}(\phi)$ theories. Phys. Rev. B 152798 (1977)

7. Sylvester, G. S.: Inequalities for Continuous-Spin Ising Ferromagnets. J. Stat. Phys. 15, 327-341 (1976)

8. Lebowitz, J. L.: Number of Phases in One Component Ferromagnets. In: Lecture Notes in Physics 80, Springer 1977

9. Griffiths, R. B.: Phase Transitions. In: Statistical Mechanics and Quantum Field Theory. Les Houches Lectures 1970. Gordon and Breach Science Publishers, New York, London, Paris 1971 